\title{
Cooperation of the Spatial Interpolation Algorithm for the Contour Map of the Shockwave Overpressure Field
}

\author{
Yongli Zhang ${ }^{1}$, Tailin Han ${ }^{1, *}$, Hong Liu ${ }^{2}$, Xiao Wang' and Enkui Zhang ${ }^{1}$ \\ ${ }^{1}$ School of Electronics and Information Engineering, Changchun University of Science and Technology, Changchun 130011, China \\ ${ }^{2}$ School of Optoelectronic Engineering, Changchun University of Science and Technology, Changchun 130011, China
}

Received 19 August 2017; Accepted 6 December 2017

\begin{abstract}
Traditionally, the peak overpressures of multi-point shockwave are obtained through the sensor array by using the electrometric method, and then the surface interpolation is carried out by the mathematical model to draw the contour lines of the shockwave overpressure field. The cross-validation method of the mean absolute error (MAE), mean relative error (MRE), and root mean square error (RMSE) was proposed in this study to achieve high precision and effective interpolation of the contour lines. The quantitative test of Kinney-Graham Formula was verified, and the peak overpressures of the multi-point shockwave of a 7.62-mm caliber gun and a naval gun were obtained through the sensor array based on polar coordinate. Then, the shockwave overpressure field was interpolated by the inverse distance weighted (IDW) interpolation, the ordinary kriging interpolation(OK), the radial basis function (RBF) interpolation, and the cubic spline (CS) function interpolation, Finally, cross-validation analysis was performed on MAE, MRE, and RMSE. Results demonstrate that the MAE of the RBF interpolation is 0.038 , and the MRE is 0.001 . The error is smallest, the accuracy is highest, and the interpolation effect is closest to the shockwave field model. The OK interpolation is close to the RBF interpolation. The MRE of the CS interpolation is 0.254 , which is relatively large. The RMSE of the IDW interpolation is 14.268 , which is abnormal. The RBF interpolation is the optimal interpolation algorithm for the shockwave overpressure field, which provides a reference for the contour drawing of the shockwave overpressure field.
\end{abstract}

Keywords: Shockwave overpressure field, Contour-map, Space interpolation, Cross-validation

\section{Introduction}

Contour lines are generated based on the spatial interpolation theory of computer graphics. For the discrete distribution and some regular geometric variable values or physical quantities, the points with the same values are interpolated and the contour lines are drawn by a mathematical method to express the physical distribution clearly and intuitively.

The service and safety performance evaluation of the shockwave overpressure field using the conventional weapon approval test is conducted to provide reliable scientific basis to assess the damage efficiency of weapons and equipment and identify the required maintenance. In the existing literature, the initial establishment of the theory of shockwave model provides the basis for the prediction of the overpressure and the drawing of the contour line of the shockwave overpressure field [1]. Previous studies showed that the test of the shockwave overpressure field to obtain the overpressure value of the sensor array at different points and draw the contour lines by the interpolation algorithm was mainly based on the electric method [2]. Interpolation compensates for the deficiency of spatial observation data points. It generates the distribution of data points with regular spacing in the range of original data distribution, according to certain grid handling method (mathematical

\footnotetext{
*E-mail address: hant1@126.com

ISSN: 1791-2377 @ 2017 Eastern Macedonia and Thrace Institute of Technology. All rights reserved. doi:10.25103/jestr.106.14
}

models) for calculation. The sensor arrays should be arranged in a Cartesian coordinate system, but the polar coordinate system had better applicability given the limited space area of the weapon [3]. The shockwave overpressure data obtained by a sensor array in a polar coordinate system showed uneven distribution, which affected the accuracy of the traditional cubic spline (CS) function interpolation and resulted in the contour error [4].

Data characteristics greatly influence the interpolation algorithm and the different optimal interpolation algorithms for different types of data [5]. In the existing literature, only the CS function interpolation was used to study the shockwave overpressure field; the error was not evaluated. The shockwave overpressure field obtained by the electrometric method has the characteristics of less measurement points, numerical changes, and sparse data, etc. Further study and evaluation of different interpolation algorithms are required to obtain high precision shockwave overpressure contour map.

The present study cites the four types of interpolation algorithm for the shockwave overpressure field on surface interpolation and the statistical method of cross-validation, which is used to compare the interpolation results to obtain the optimal interpolation algorithm.

\section{State of the art}

The different types of shockwave overpressure fields have been tested in recent years. The improvement of test 
technology and computer simulation capability guarantees the accuracy of the overpressure peak and the further understanding of shockwave physics model. Zhao Haitao et al. programmed the air explosion numerical simulation program and compared it with the commercial software AUTODYN. The air blast shockwave propagation was conducted to verify the empirical formula, but the shockwave overpressure field information was not analyzed [6]. Zhang Yuanping performed the near-ground point source explosion test of the single direction of 11 points through a sensor array by using the electrometric method, and deduced the TNT equivalent by curve fitting with different point pressure values through the empirical formula,. However, the similarity measurement precision and other angles were not explained [7]. The multi direction test of the near-ground point source explosion was conducted by Feng Hao to obtain a single quadrant peak overpressure of shockwave, through the improvement of the polar form and lay out of non-uniform angles and different radii of 15 test points. The contour lines were drawn with CS function interpolation, according to the similarity law. However, the interpolation precision was not studied [4]. Wang Yang et al. analyzed the physical model of the point source explosion and muzzle shockwave of heavy-caliber weapons, and obtained the initial and the overpressure peak contour through numerical simulation. However, the ground reflection and far-field condition of shockwave were not considered [8]. Lai Fuwen et al. tested the medium and far field shockwave of heavy-caliber weapons (naval gun) through the sensor array in a polar coordinate system, obtained the 25 pointes peak overpressures, and plotted the shockwave overpressure contour by cubic spline interpolation. However, the interpolation error was not quantified [3]. The shockwave of minor-caliber weapons was simulated and tested to obtain the corresponding characteristics of shockwave propagation by Hristov, but the effects of different interpolation algorithms on the shockwave contour were not analyzed [9]. Rehman simulated the pulse noise of the weapon to validate the wave propagation characteristics, and provided a reference for the contour plot in the shockwave far field [10].

Data types and numbers have the greatest impact on the effect of interpolation algorithm and contour drawing, especially the number of value points $[11,12]$. Tugrul et al. studied the inverse distance weighted (IDW) interpolation algorithm, which eliminated the duck egg effect with different weighting coefficients. However, the data type influence was not analyzed further [13, 14]. Kerry and Roger introduced the principle of kriging interpolation algorithm and verified the application of different algorithms, but the effects of number were not sufficiently studied $[15,16]$. Pouderoux and Dehghan conducted a deep research on the radial basis function (RBF) interpolation and smoothing, introduced the steps for two-dimensional interpolation, and pointed out that the RBF interpolation had the best effect, thereby providing a reference for the interpolation algorithm [17-19]. The settlement of foundation ditch was analyzed based on the fifteen observation points by using the IDW interpolation, kriging interpolation, and Shepard interpolation. The cross-validation result showed that the accuracy of the IDW interpolation is the highest [20]. However, this conclusion is not accurate for the shockwave overpressure field, because the overpressure value of the shockwave and the settlement volume are different from the single point source influence data. Cheng Peng used the diurnal temperature of twenty-seven weather stations to interpolate the annual average temperature of the Shanxi plateau by eight types of spatial interpolation, including inverse distance weighting, global polynomials, local polynomials, radial basis functions, spline functions, trend surfaces, ordinary kriging, and cooperative kriging. Precision comparison was carried out by using one cross validation. The radial basis function and the ordinary Kerri interpolation have the highest precision [21]. The daily temperature is mainly affected by the sun, and the shockwave is the one-point source data, which provide reference for the shockwave overpressure field interpolation. The interpolation algorithms of ordinary kriging, cubic spline, and inverse distance weighted were compared and analyzed based on geostatistics, mathematical functions, and geometric methods. If sparse data were available, they were used for ordinary kriging (OK) interpolation. The interpolation precision of cubic spline function was high when the more and uniform data were available [22]. However, the impact of quantity was not analyzed and quantified.

In the existing literature, the shockwave overpressure prediction and near-field shockwave model and interpolation of the other physical quantity have been studied. However, few studies on surface interpolation for sparse data of shockwave overpressure peaks are available, especially the evaluation of interpolation accuracy of different interpolation algorithms.

The present study proposes the evaluation method of cross validation and obtains multipoint overpressure value of different types of shockwave field, such as the near-ground point source explosion, minor-caliber weapons, and naval gun. The overpressure peaks are interpolated by the IDW interpolation, the $\mathrm{OK}$ interpolation, the $\mathrm{RBF}$ interpolation, and the CS function interpolation. The merits and demerits of different interpolation algorithms are compared based on the shockwave model and error evaluation to provide the basis for the contour plot of the shockwave overpressure field.

The remainder of this study is organized as follows. Section 3 presents the tests of the multipoint overpressure values of three types of shockwave field, analyzes the four types of interpolation algorithms, and proposes the evaluation method for cross validation. Section 4 presents the surface interpolation of the three types of shockwave fields, drawings of the contour-maps of the different algorithms, and the contrast between the effects and errors. Section 5 presents the summary of the conclusions.

\section{Methodology}

The data of the near-ground point source explosion, minorcaliber weapons, and naval gun obtained by the electrometric method and processed by data filtering are different.

The interpolation method can be divided into two categories, according to its mathematical principles, as follows: the deterministic interpolation and the geostatistical interpolation. The deterministic interpolation method is based on the appearance of the interior region or on its smoothness, and the surface is created by the known samples. Geostatistical interpolation can not only quantify the spatial autocorrelation among known points, but also explain the spatial distribution of sampling points in the predicted area by using the statistical characteristics of known samples [11]. 
Yongli Zhang, Tailin Han, Hong Liu, Xiao Wang and Enkui Zhang/

Journal of Engineering Science and Technology Review 10 (6) (2017) 104-110

The four types of interpolation algorithms used in this study belong to geostatistical interpolation, such as IDW interpolation, RBF interpolation, CS function interpolation, and $\mathrm{OK}$ interpolation.

\subsection{Shockwave overpressure field data}

Blast wave is a spherical wave of isotropy at rest and expanding outward. The overpressure value is calculated at different distances, with the standard explosive ball exploding in the air, using the theoretical or empirical formula. The much accurate calculation of the overpressure explosion of TNT explosive ball in the air is the KinneyGraham Formula, as follows:

$$
\begin{aligned}
\frac{\Delta p_{\max }}{p_{\text {air }}}=\frac{808\left[1+\left(\frac{f_{d} \bar{R}}{4.5}\right)^{2}\right]}{\sqrt{1+\left(\frac{f_{d} \bar{R}}{0.488}\right)^{2}} \sqrt{1+\left(\frac{f_{d} \bar{R}}{0.32}\right)^{2}} \sqrt{1+\left(\frac{f_{d} \bar{R}}{1.35}\right)^{2}}} \\
f_{d}=\sqrt[3]{\frac{p_{\text {air }}}{p_{\text {0air }}} \frac{T_{\text {oair }}}{T_{\text {air }}}} \\
\bar{R}=r / \sqrt[3]{\omega}
\end{aligned}
$$

where $f_{d}$ is the factor of atmospheric transmission, $p_{\text {air }}$ is the atmospheric pressure at the test site $\left(\mathrm{kg} / \mathrm{m}^{2}\right), p_{0 a i r}$ is the standard atmosphere $\left(1.03323 \mathrm{~kg} / \mathrm{m}^{2}\right), T_{\text {air }}$ is the atmospheric temperature at the test site $(K), T_{0 \text { air }}$ is the standard atmosphere temperature $(288.16 K), \bar{R}$ is the ratio of distance $\left(m / \mathrm{kg}^{1 / 3}, 0.053 \leq \bar{R} \leq 500\right), r$ is the distance between the test point and the core $(m)$, and $\omega$ is the TNT equivalent of explosives $(\mathrm{kg})$.

The explosion shockwave model has been proven, and the TNT equivalent has been introduced [7]. The overpressure empirical equation (Eq. (1)) is used to model the explosion shockwave in the infinite space, and the overpressure value is calculated in the range of approximately 13-30 $\mathrm{m}$ from the distance to the core. The arbitrary direction is $0^{\circ}$ when the core is considered the center, and five radial points are taken at the radial equal intervals of $30^{\circ}, 60^{\circ}, 90^{\circ}, 120^{\circ}$ and $150^{\circ}$. The blast wave is isotropic, and the data on the same radius are equal. The overpressure peaks of $10,14,18,22$, and $26 \mathrm{~m}$ were 168.0, $78.4,46.1,31.3$, and $23.2 \mathrm{kPa}$, respectively. A total of 25 type value points are considered.

The weapon muzzle shockwave is a spherical shockwave with a directional characteristic. The overpressure contour is peach-shaped, and its intensity is the strongest along the propagation direction [8]. The different weapon characteristics and launching conditions obtained different shockwave results. The shockwave field presented by a naval gun is much complicated given the influence of secondary flame and ground overpressure [3].

The sensor array is arranged on one side when the shockwave is tested, considering the left and right symmetry of the muzzle. The overpressure peaks of a naval gun and $7.62-\mathrm{mm}$ caliber gun are obtained in polar form, as shown in Tables 1 and 2, respectively. The firing direction of a naval gun is $0^{\circ}$, and the range of $120^{\circ}$ to $240^{\circ}$ is selected; it is distributed every $30^{\circ}$. The test radial range is $3-7 \mathrm{~m}$, and the placement interval is $1 \mathrm{~m}$. Five points are placed on the same radial direction, and, thus, a total of 25 points are placed. A 7.62-mm caliber gun is placed on the direction of $0^{\circ}$, and the test point is at the origin of the muzzle. The interval between adjacent points is $22.5^{\circ}$, and the distance between adjacent measuring points in the radial direction is $0.25 \mathrm{~m}$. Four points are placed on the same radial direction.

\begin{tabular}{|c|c|c|c|c|c|}
\hline Data & $r=3 \mathrm{~m}$ & $r=4 m$ & $r=5 \mathrm{~m}$ & $r=6 \mathrm{~m}$ & $r=7 \mathrm{~m}$ \\
\hline$\theta=30^{\circ}$ & 20.3 & 15.6 & 11.4 & 9.3 & 7.62 \\
\hline$\theta=60^{\circ}$ & 15.1 & 12.5 & 9.7 & 8.7 & 7.4 \\
\hline$\theta=90^{\circ}$ & 11.0 & 8.7 & 6.0 & 3.9 & 3.5 \\
\hline$\theta=120^{\circ}$ & 7.0 & 8.4 & 6.4 & 4.2 & 3.7 \\
\hline$\theta=150^{\circ}$ & 4.6 & 3.8 & 3.9 & 3.1 & 3.0 \\
\hline
\end{tabular}
Thus, a total of 28 points are placed.

Table 1. Peak overpressure data of a naval gun shockwave

Table 2. Peak overpressure data of a 7.62-mm gun shockwave $(\mathrm{kPa})$

\begin{tabular}{l|l|l|l|l}
\hline Data & $r=0.25 m$ & $r=0.5 m$ & $r=0.75 m$ & $r=1.0 m$ \\
\hline$\theta=22.5^{\circ}$ & 47.41 & 18.25 & 10.73 & 6.96 \\
$\theta=45^{\circ}$ & 41.86 & 14.69 & 9.10 & 5.67 \\
$\theta=67.5^{\circ}$ & 29.91 & 10.32 & 5.81 & 3.76 \\
$\theta=90^{\circ}$ & 16.76 & 7.30 & 4.55 & 3.06 \\
$\theta=112.5^{\circ}$ & 10.66 & 4.34 & 2.88 & 2.36 \\
$\theta=135^{\circ}$ & 5.76 & 3.37 & 2.10 & 1.56 \\
$\theta=157.5^{\circ}$ & 4.01 & 2.27 & 1.26 & 0.87 \\
\hline
\end{tabular}

\subsection{IDW interpolation method}

The basic principle of the IDW interpolation method is that a series of discrete points is distributed in the plane, and its position and attribute values are known as $\left(x_{i}, y_{i}\right)$ and $z_{i}(i=1,2, \mathrm{~L} n)$. The value of point $P$ is evaluated by the distance weighted interpolation based on the property values of the discrete point $p(x, y)$, which is an arbitrary grid node. The influence of the surrounding points on point $P$ is different from the difference in the distribution location of point $P(z)$, which is called the weight function $\omega_{i}(x, y)$, as follows:

$$
W_{i}(x, y)=1 /\left[d_{i}(x, y)\right]^{u}
$$

where $d_{i}(x, y)=\sqrt{\left(x-x_{i}\right)^{2}+\left(y-y_{i}\right)^{2}}$ is the distance from point $\left(x_{i}, y_{i}\right)$ to point $P(x, y)$.

The square parameter controls the weight coefficient and decreases with the increase in the grid node distance. When calculating a grid node, the weight of a specific data point is proportional to the reciprocal of the distance from the point of the specified square to the observation point. The weight function is mainly related to distance, and usually to the direction. If the point is taken evenly on four directions around point $P$, then the direction factor is not considered. Thus, the value of the interpolation point $P(z)$ is obtained as follows:

$$
P(z)=\sum_{i=1}^{n} \frac{z_{i}}{\left[d_{i}(x, y)\right]^{u}} / \sum_{i=1}^{n} \frac{1}{\left[d_{i}(x, y)\right]^{u}}
$$

Allocation of the number of decision values of the weight function. 
Yongli Zhang, Tailin Han, Hong Liu, Xiao Wang and Enkui Zhang/

Journal of Engineering Science and Technology Review 10 (6) (2017) 104-110

For a larger square, the closer data points are provided with higher weights, or the weights are evenly distributed to each point. Therefore, different values greatly influence point $P(z)$. In addition, the calculation values are easily affected by the data point cluster. The results often show an isolated point, and the value is significantly higher than the surrounding data points. This approach is known as the "duck egg" distribution pattern.

\subsection{Kriging interpolation method}

Kriging interpolation space, also called the best autocovariance interpolation, is a method of optimal, linear, and unbiased estimation for variable values in the unsampled region that uses the original data of the regionalized variables and the structural features of the variation functions. The value of the unknown sample points, which are determined by the weighting of the known sample points, is expressed as follows:

$$
Z(x)=\sum_{i=1}^{n} w_{i} Z\left(x_{i}\right)
$$

where $Z(x)$ is the value of an unknown sample point, $Z\left(x_{i}\right)$ is the value of the known sample points around the unknown sample point, $w_{i}$ is the weight of a known sample point $i$ to the unknown sample point, and $n$ is the number of the known sample points.

When weights are assigned with values, kriging interpolation not only considers the distance, but also the spatial relationship between known and unknown sample points through the variation function and structural analysis, which is different from the IDW interpolation. Many types of kriging interpolation methods are available. The traditional kriging method is often used when the expectation is unknown. In this method, the data are assumed to change into normal distribution. The interpolation process is similar to the weighted sliding average, and the weight is determined through the variation function, which is often referred to as local optimal linear unbiased estimation. kriging interpolation method commonly uses semi-variant function models such as Gaussian, linear, spherical, and exponential models. It has different applications in different areas.

\subsection{RBF interpolation}

The RBF interpolation is a combination of multiple data interpolation methods. The interpolation principle can be expressed as follows:

For the set $X=\left\{X_{i} \in R^{d}, i=1,2, \mathrm{~L}, N\right\}$ of $\mathrm{N}$ known sampling points and the corresponding set $Y=\left\{f_{i} \in R^{d}, i=1,2, \mathrm{~L}, N\right\} \quad$ of $\mathrm{N}$ real numbers, an interpolation function that satisfies the interpolation conditions as follows is determined:

$$
s\left(X_{i}\right)=f_{i}, i=1,2, \mathrm{~L}, N
$$

where $\left\{\phi\left(\left\|X-X_{i}\right\|\right) \mid i=1,2, \mathrm{~L}, N\right\}$ is a collection of $\mathrm{N}$ radial basis functions. The known point $X_{i} \in R^{d}, i=1,2, \mathrm{~L}, N$ is the center of the radial basis function. $w_{i}(i=1,2, \mathrm{~L}, N)$ is the unknown power coefficient. All the RBF interpolation methods are accurate interpolators and can be used to all data. A slip coefficient is introduced in the base function to generate a much rounded surface. Some usual basic functions, such as thin strip spline function, multiple quadratic function, and natural CS function, are available.

\subsection{CS interpolation method}

The spline function is a piecewise function that fits only a few points each time, while the connection of the curve segment is continuous. Therefore, the surface curvature is minimized by using the sample points to fit the smooth curve $[23,24]$. The CS function is not only smooth, but can also guarantee the continuity of the first and second derivatives of the fitting curve.

The set of data points are as follows:

$$
\left(x_{0}, y_{0}\right),\left(x_{1}, y_{1}\right), \mathrm{L},\left(x_{\mathrm{n}}, y_{n}\right)
$$

where $x_{0}<x_{1}<\mathrm{L}<x_{n}$. A function to satisfy the condition is determined, as follows:

1) $S(x)=y_{i},(i=0,1,2, \mathrm{~L} n)$;

2) Within the range $\left[x_{0}, x_{n}\right], S(x)$, second order is read and guided;

3) Within each mini zone, $S(x)$, is the cubic polynomial of variable $x$.

Then, $S(x)$ is the CS function or CS polynomial for the data points.

In the following set of data points: $\left(x_{i}, y_{i}\right), i=1,2, \mathrm{~L}, n$, $m_{j}$ is the slope of $\left(x_{i}, y_{i}\right)$. Then, the CS interpolation function expression for section $j$ is as follows:

$$
y_{j}(x)=a_{j} x^{3}+b_{j} x^{2}+c_{j} x+d_{j} \quad j=1,2, \mathrm{~L}, n
$$

and $h_{j}=x_{j}-x_{j-1}$. The interpolation function coefficient $a_{j}$, $b_{j}, c_{j}, d_{j}$ is obtained as follows:

$$
\begin{aligned}
& a_{j}= \frac{m_{j}+m_{j-1}}{h_{j}^{2}}-\frac{2\left(y_{j}-y_{j-1}\right)}{h_{j}^{3}} \\
& b_{j}= \frac{-m_{j}\left(x_{j}+2 x_{j-1}\right)+m_{j-1}\left(2 x_{j}+x_{j-1}\right)}{h_{j}^{2}} \\
&+\frac{3\left(y_{j}-y_{j-1}\right)\left(x_{j}+x_{j-1}\right)}{h_{j}^{3}} \\
& c_{j}= \frac{m_{j-1} x_{j}\left(x_{j}+2 x_{j-1}\right)-m_{j-1} x_{j-1}\left(2 x_{j}+x_{j-1}\right)}{h_{j}^{2}} \\
&-\frac{6\left(y_{j}-y_{j-1}\right) x_{j} x_{j-1}}{h_{j}^{3}} \\
& d_{j}=\frac{\left(m_{j} x_{j-1}+m_{j-1} x_{j}\right) x_{j} x_{j-1}}{h_{j}^{2}} \\
&+\frac{y_{j} x_{j-1}^{2}\left(3 x_{j}-x_{j-1}\right)+y_{j-1} x_{j}^{2}\left(x_{j}-3 x_{j-1}\right)}{h_{j}^{3}}
\end{aligned}
$$

Although the spline interpolation speed is fast and has better visual effect, a large error is obtained in the area estimation of the large variation of the attribute in short distance. 


\subsection{Cross Validation}

The overpressure data error is analyzed by using the cross validation method, which can be used for all measuring points, assuming that the data is unknown. For the remaining point data, a different interpolation method is used to compute the simulation value by combining the calculating error of the observed value [25-27].

The error evaluation method mainly adopts the mean absolute error (MAE), mean relative error (MRE), and root mean square error (RMSE). MAE reflects the error range of the simulated value, MRE reflects the accuracy of the simulated value for the observed value, and RMSE reflects the sensitivity and extreme value of the simulated value.

$$
\begin{aligned}
& M A E=\frac{1}{n} \sum_{i=1}^{n}\left|Z_{i}-Z_{i}^{\prime}\right| \\
& M R E=\frac{1}{n} \sum_{i=1}^{n}\left|\frac{Z_{i}-Z_{i}^{\prime}}{Z_{i}}\right|
\end{aligned}
$$

$$
R M S E=\sqrt{\frac{1}{n} \sum_{i=1}^{n}\left(Z_{i}^{\prime}-Z_{i}\right)^{2}}
$$

where $Z_{i}$ is the actual measured value of point $i, Z_{i}^{\prime}$ is the simulation value of point $i$, and $n$ is the number of points.

\section{Result Analysis and Discussion}

\subsection{Drawing contour map}

The best interpolation parameters were selected for intuitive comparison of the interpolation results of the four methods for the three types of shockwave overpressure peak data in the study. IDW uses the third order equation. OK selects the linear transformation. RBF uses thin plate spline function, and $\mathrm{CS}$ adopts the three bending moment method. The wave overpressure peak fields of the four different models are shown in Figs. 1, 2, and 3.
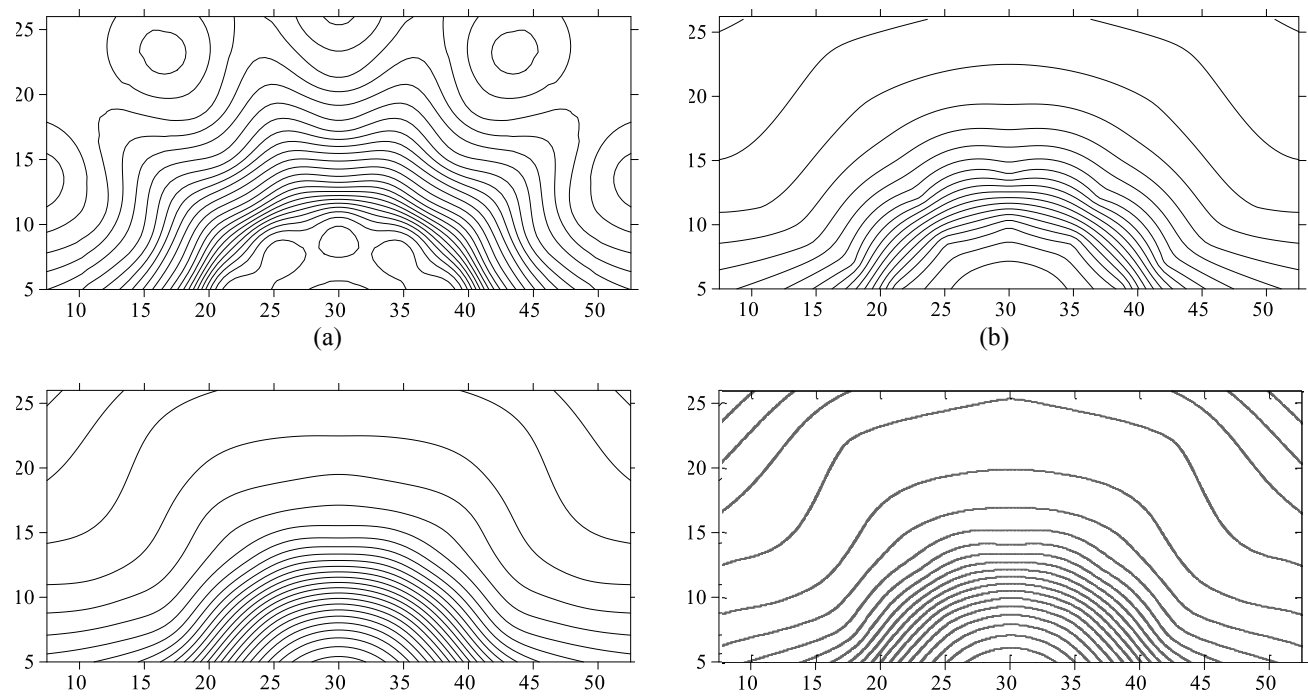

(c)

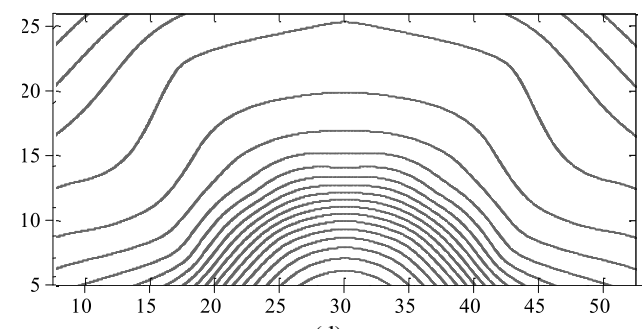

(d)

Fig. 1. Contour-map of the blast (a) under IDW method, (b) under OK method, (c) under RBF method, and (d) under CS method.

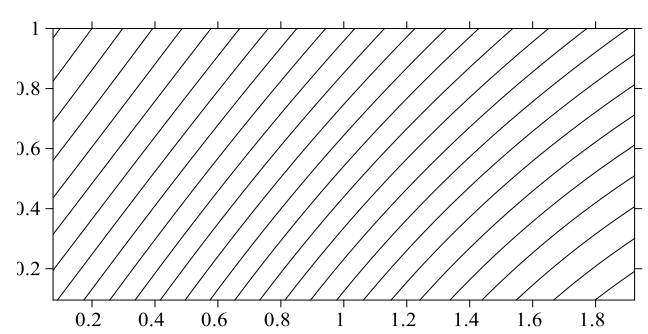

(a)

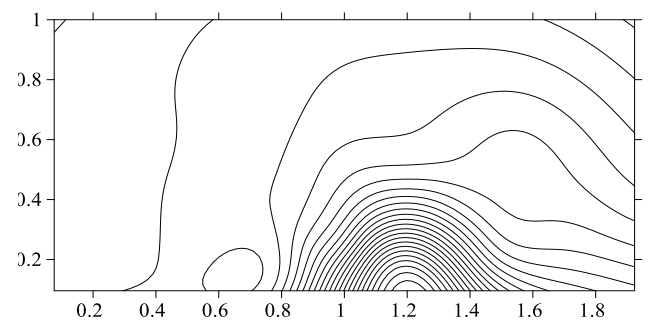

(c)

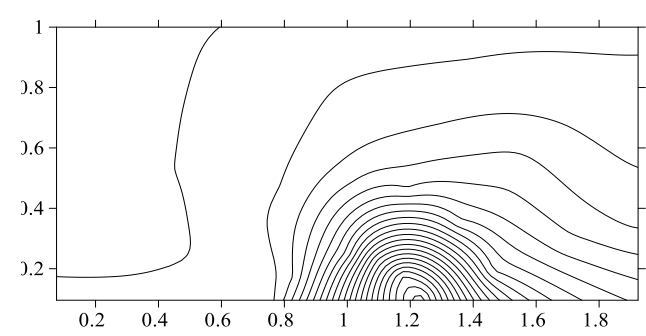

(b)

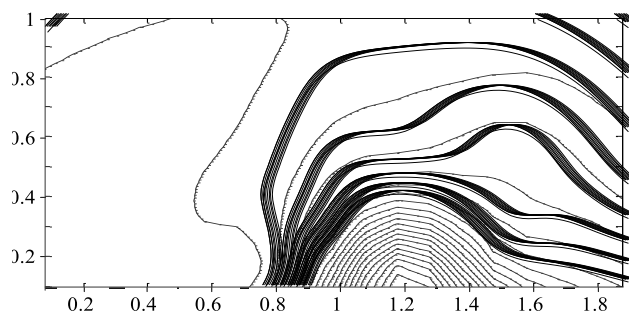

(d)

Fig. 2 Contour map of a 7.62-mm caliber gun (a) under the IDW method, (b) under the OK method, (c) under the RBF method, and (d) the CS method. 

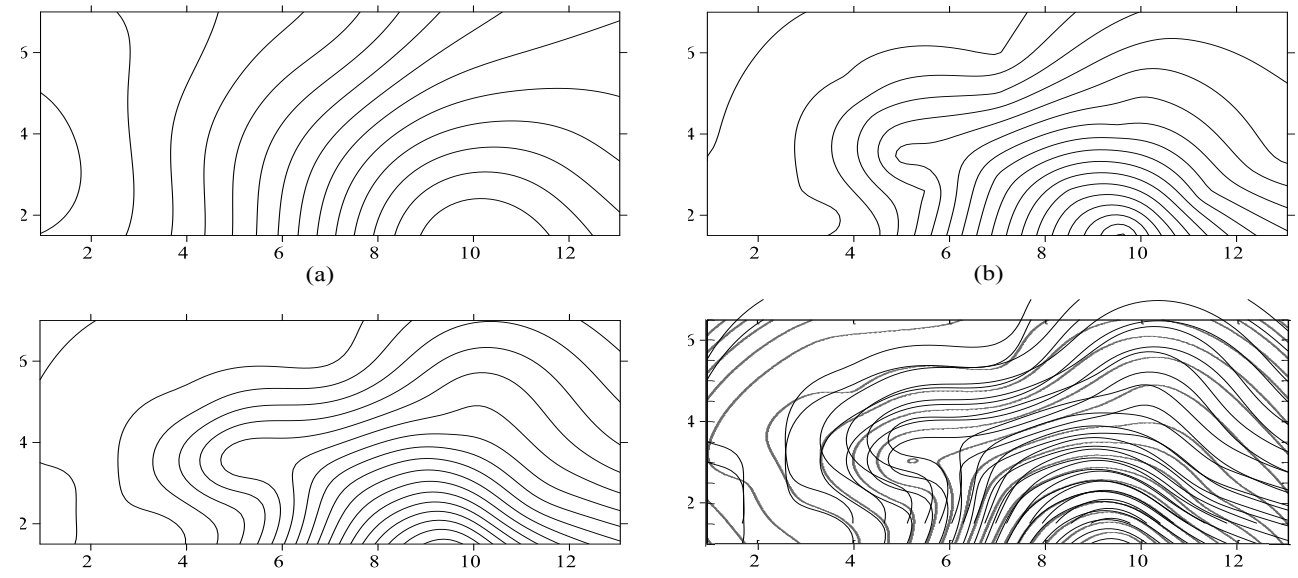

(c)

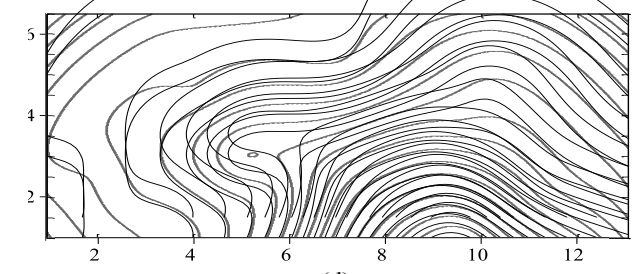

(d)

Fig. 3. Contour map of a naval gun (a) under the IDW method, (b) under the OK method, (c) under the RBF method, and (d) under the CS method.

The blast shockwave field has the same centrality, and the overpressure value decreases with increasing radius. Fig. 1 shows that the contour lines generated by the four methods can reflect the general situation of the wave diffusion of the point burst model. The super pressure contour density is high when the shockwave field is closer to the detonation. The direction of $0^{\circ}$ and $180^{\circ}$ is negatively influence by the number of points and the placement. The contour line, which produces an extreme value near the explosion and the far field, experienced the duck egg effect. The concentricity intensified with the increase in the radius, as generated by the IDW method, which does not accord with the law of infinite space propagation of point explosion. The contour line becomes sparse near the bursting core less than the midfield, and the midfield contour fluctuates. The contour generated by the IDW method is easily affected by the boundary value, and the smoothing effect becomes slightly worse. The RBF and CS methods have similar interpolation effect. However, the RBF method is slightly richer and more concentric.

The muzzle shockwave overpressure field has the characteristics of directivity and dynamic circle. As shown in Fig. 2, in addition to the IDW method, the contour lines point to the $0^{\circ}$ direction, and the contour center deviation is approximately 0.2 . The other three methods reflect the characteristics of the chamber shockwave. The IDW method produces the anomaly of contour line, and the contour lines produced by the $\mathrm{OK}$ method points to the horizontal direction of $180^{\circ}$. The midfield in the direction of $90^{\circ}$ fluctuates, and the contour lines produced by RBF are the most rapidly attenuated near the center. The contour line is relatively smooth, and the effect is better. Finally, the contour line produced by the CS method is abnormal in the direction of $135^{\circ}$.

Fig. 3 shows that the contour lines produced by four methods reflect the influence of weapon caliber on the muzzle shockwave, that is, the particularity of the shockwave field is mainly reflected on the middle and far fields. The contour line is affected by the surface reflection. A great difference between the IDW method and the other three algorithms is observed, and an anomaly appears on the contour in the direction of $80^{\circ}$. The OK, RBF, and CS methods have the same change rules; only the smoothness and elevation changed slightly on the attenuation in the direction of $120^{\circ}$.

The comparative results of the interpolation effect of the three different types of shockwave fields show that, in general, the RBF method has the best effect, and the CS method interpolation effect is extremely close. The OK method has some fluctuations, and the IDW method is abnormal.

\subsection{Error analysis discussion}

The MAE, MRE, and RMSE are calculated, and the error between the estimated value and type of value point of each method is analyzed based on the intuitionistic comparison of the contour map. The MAE, MRE, and RMSE of the blast, the 7.62-mm caliber gun, and the naval gun are compared under the four interpolation methods, as shown in Tables 3, 4, and 5. Table 3 shows that for MAE, MRE, and RMSE, RBF $<\mathrm{OK}<\mathrm{CS}<\mathrm{IDW}$. Thus, under the $\mathrm{RBF}$ method, the interpolation error is minimal. Under the OK method, the interpolation is relatively close. Under the CS method, the interpolation error is slightly large. Under the IDW method, the interpolation error is the largest. The two errors of the RBF and the OK methods are relatively close, and the difference in MRE is only 0.007, indicating that the interpolation effect is similar. The RMSE of the IDW method interpolation is large, indicating that the IDW method is abnormal. The statistics in Tables 4 and 5 is consistent with Table 3 , and the regularity is stronger.

Overall, the analysis conclusion of the cross validation error is that the RBF method interpolation effect is best, followed by the OK method interpolation. The CS method interpolation error is slightly large, and the IDW method interpolation is abnormal.

Table 3. Cross validation error of the interpolation of blast

\begin{tabular}{l|l|l|l}
\hline Data & MAE & MRE & RMSE \\
\hline IDW & 11.371 & 0.181 & 14.268 \\
OK & 0.664 & 0.008 & 0.964 \\
RBF & 0.038 & 0.001 & 0.056 \\
CS & 9.627 & 0.186 & 11.439 \\
\hline
\end{tabular}

Table 4. Cross validation error of the interpolation of a 7.62-mm caliber gun

\begin{tabular}{l|l|l|l}
\hline Data & MAE & MRE & RMSE \\
\hline IDW & 9.434 & 2.111 & 13.504 \\
OK & 0.093 & 0.009 & 0.159 \\
RBF & 0.008 & 0.001 & 0.013 \\
CS & 1.760 & 0.254 & 2.771 \\
\hline
\end{tabular}

Table 5. Cross validation error of the interpolation of a naval gun

\begin{tabular}{l|l|l|l}
\hline Data & MAE & MRE & RMSE \\
\hline IDW & 2.187 & 0.347 & 2.794 \\
OK & 0.030 & 0.005 & 0.047 \\
RBF & 0.002 & 0.001 & 0.003 \\
CS & 0.798 & 0.148 & 1.122 \\
\hline
\end{tabular}




\section{Conclusions}

To draw high precision and effective shockwave overpressure field contour, an error evaluation method was established to evaluate the interpolation effect of four interpolation methods, including IDW, OK, RBF, and CS for the overpressure peak field of different types of shockwaves of the blast, 7.62-mm caliber gun, and naval gun. The MAE, MRE, and RMSE of the four interpolation results were cross-checked and compared. The following conclusions could be drawn:

1) The contour map shows that the RBF, OK, and CS methods reflected the overall change in the overpressure peak field. The RBF method highlights the details, followed by the OK and the CS methods. However, the IDW method cannot evidently reflect the change in the overpressure peak field.

2) The MAE, MRE, and RMSE results are analyzed, according to the error evaluation method. The three types of errors of the RBF method interpolation are the least, and that of the IDW method are the largest. The RBF method is superior to the CS method. The error of the RBF and OK methods is close to that of the 7.62-mm caliber gun and the naval gun shockwave field. Thus, the interpolation precision can satisfy the requirement.

3) The RBF method interpolation is the optimal interpolation algorithm in the overall analysis.

Thus, the spatial interpolation algorithm is introduced in the shockwave field to improve the precision of the pressure line drawing, such as the shockwave field of the electrometric method. It has a certain reference to the perfection of the shockwave overpressure field model. The data present two dimensional characteristics because the shockwave overpressure sensor is located in the same horizontal plane height. Therefore, in future studies, changing the different placement heights will result in much accurate contours of the shockwave overpressure field. Additional influencing factors should be considered in the future.

This is an Open Access article distributed under the terms of the Creative Commons Attribution Licence

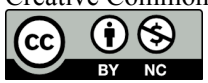

\section{References}

1. K. Fansler, "Description of muzzle blast by modified ideal scaling models". Shock and Vibration, 5(1), 1998, pp.1-12.

2. R. Stoughton, "Measurements of small-caliber ballistic shock waves in air". Acoustical Society of America Journal, 102(2), 1997, pp.781-787.

3. Lai Fuwen, Zhang Zhijie, Hu Guimei, Zhang Jianyu, "A Method to Measure Muzzle Shockwave Pressure Field for a Naval Gun". Chinese Journal of Sensors and Actuators, 28(1), 2015, pp.77-80.

4. Feng Hao, Zhang Zhijie, "Reconstruction of shockwave pressure field based on distributed test system". Journal of Measurement Science and Instrumentation, 6(1), 2015, pp.25-29.

5. Luo Yiyong, Zhang Liting, Chen Zuan, "Precision analysis of data gridding and calculation of volume based on surfer". Science of Surveying and Mapping, 34(5), 2009, pp.97-99.

6. Zhao Haitao, Wang Cheng, "High Resolution Numerical Simulation of Air Explosion". Acta Armamentarii, 34(12), 2013, pp.15361546.

7. Zhang Yuanping, Chi Jiachun, Gong Yanqing, Wang Guangjun, "Measure technology of blast shockwave pressure and disposal method of complicated signal". Chinese Journal of Scientific Instrument, 28(8), 2007, pp.324-327.

8. Wang Yang, Jiang Xiaohai, Gguo Zeqing, "Numerical Analysis on Physical Model of Muzzle Blast Wave". Journal of Ballistics, 22(1), 2010, pp.57-60.

9. N Hristov, A Kari, D Jerković, S Savić, R Sirovatka, "Simulation and Measurements of Small Arms Blast Wave Overpressure in the Process of Designing a Silencer". Measurement Science Review, 15(1), 2015, pp.27-34.

10. Rehman, H., Hwang, S.H., Fajar, B. et al., "Analysis and attenuation of impulsive sound pressure in large caliber weapon during muzzle blast". Journal of Mechanical Science and Technology, 25(10), 2015, pp.2601-2606.

11. J Chai, T Miyoshi, E Nakamae. "Contour Interpolation and Surface Reconstruction of Smooth Terrain Models". In: Proceedings of the 1998 IEEE symposium on Volume visualization, North Carolina, USA: IEEE, 1998, pp.27-33.

12. Hu Yaofeng, H Lin, P Zhang, "Research on key algorithm of automatic contour interpolation". Science of Surveying \& Mapping, (8), 2016, pp.24-27.

13. B Tugrul, H Polat, "Privacy-Preserving Inverse Distance Weighted Interpolation". Arabian Journal for Science \& Engineering, 39(4), 2014, pp.2773-2781.

14. Z. Li, Y. Wu. "Inverse distance weighted interpolation involving position shading". Acta Geodaetica Et Cartographica Sinica, 44(1), 2015, pp.91-98.
15. Kerry, K., Hawick, K. "Kriging interpolation on high-performance computers". In: Proceedings of the International Conference and Exhibition on High-Performance Computing and Networking, The Netherlands, Amsterdam: Springer, 1998, pp.429-438.

16. Roger Woodard, "Interpolation of Spatial Data: Some Theory for Kriging”. Technimetrics, 42(4), 2012, pp.436-437.

17 GR Liu, L Yan, JG Wang, YT Gu, "Point interpolation method based on local residual formulation using radial basis functions". Structural Engineering \& Mechanics, 14 (6), 2002, pp.713-732.

18. J Pouderoux, JC Gonzato, I Tobor, P Guitton. "Adaptive hierarchical RBF interpolation for creating smooth digital elevation models". In: GIS'04, Washington DC, USA: ACM, 2004, pp.232240 .

19. M Dehghan, A Ghesmati. "Numerical simulation of twodimensional sine-Gordon solutions via a local weak meshless technique based on the radial point interpolation method (RPIM)". Computer Physics Communications, 181 (4), 2010, pp.772-786.

20. Wang Sanjun, Hu Xianhui, "Study on Selection of Settlement Interpolation Method for Sparse Monitoring Points". Beijing Surveying and Mapping, (3), 2017, pp.71-74.

21. Cheng Peng, Ren Hongrui, "Spatial Interpolation Analysis of Annual Average Air Temperature in Shanxi Plateau". Journal of Hebei Normal University: Natural Science Edition, 40(1), 2016, pp.73-79.

22. Liu Guangmeng, Wang Yunjia, Zhang Hairong, Wang Dong, "Comparative Study of Several Interpolation Methods on Spatial Analysis". Geomatics World, (3), 2011, pp.41-45.

23. C Zhu, "The Calculation Principle of Double Three Spline Interpolation Function and Its Application". Engineering of Surveying \& Mapping, 7(2), 1998, pp.17-21.

24. B Ren, H Liu, L Yang, L Cheng, "Smooth support vector regression based on modification spline interpolation". Journal of Theoretical and Applied Information Technology, 40(1), 2012, pp.92-100.

25. Song Liqiong, Tian Yuan, Wu Lun, Zhang Hui, "On Comparison of Spatial Interpolation Methods of Daily Rainfall Data: A case Study of Shenzhen". Geo-Information Science, 10(5), 2008, pp.566-572.

26. G Wahba, J Wendelberger, "Some New Mathematical Methods for Variational Objective Analysis Using Splines and Cross Validation”. Monthly Weather Review, 108(8), 1980, pp.1122-1143.

27. TT Wong, "Performance evaluation of classification algorithms by $\mathrm{k}$ -fold and leave-one-out cross validation". Pattern Recognition, 48(9), 2015, pp.2839-2846. 